\title{
Qualidade do solo em sistemas de produção de hortaliças orgânico e convencional
}

\author{
Pedro J Valarini' ${ }^{1}$ Fernanda RA Oliveira ${ }^{2}$; Sônia de Fátima Schilickmann ${ }^{3}$; Ronei J Poppi ${ }^{4}$ \\ ${ }^{1}$ Embrapa Meio Ambiente, C. Postal 69, 13820-000 Jaguariúna-SP; valarini@cnpma.embrapa.br; ${ }^{2}$ UFSCar, SP-330, km 174, 13600-970 \\ Araras-SP; ${ }^{3}$ COAGRIS Estrada da Capivara, 379, Aguassai, 06715-725 Cotia-SP; ${ }^{4}$ UNICAMP, Inst. Química C. Postal 6154, 13084-971 \\ Campinas-SP
}

\begin{abstract}
RESUMO
Sendo o solo um dos principais pilares de sustentabilidade de todo sistema de produção, a análise integrada dos atributos edafobiológicos pode constituir-se em ferramenta importante para avaliar a qualidade do mesmo. O objetivo do trabalho foi avaliar a qualidade do solo em função das práticas agrícolas adotadas, mediante a análise integrada de atributos físicos, químicos e biológicos. A pesquisa foi realizada em 2006 e 2007 nas microrregiões de Ibiúna e Socorro, no estado de São Paulo, em pequenas propriedades familiares orgânicas e convencionais. Foram avaliados os atributos físicos, químicos e biológicos, mediante coletas de amostras de solos sob cultivo de hortaliças e em solos de mata ou pastagem. O levantamento de dados sobre as práticas agrícolas foi realizado em cada sistema de produção. Os dados obtidos foram submetidos à análise de componentes principais (ACP). A ACP identificou maior grau de similaridade entre os solos sob cultivo em Socorro e suas respectivas testemunhas que os solos de Ibiúna, nitidamente separados em função do seu uso (mata ou cultivo) e indicando melhor manejo dos mesmos em Socorro. De maneira geral, concluiu-se que as práticas agrícolas utilizadas na maioria das propriedades orgânicas e convencionais favoreceram a degradação do solo, devida principalmente ao revolvimento intensivo e à ausência de cobertura do solo. Estes fatores provocaram redução dos teores de matéria orgânica do solo, da biomassa microbiana, da emergência de plântulas e da estabilidade de agregados nas áreas de cultivo em relação às áreas testemunhas.
\end{abstract}

Palavras-chave: atributos físicos, atributos químicos, atributos biológicos, solo, práticas de manejo, produtor familiar.

\begin{abstract}
Soil quality in organic and conventional vegetables production systems
\end{abstract}

The soil is one of the most important sustainability columns of the production system. The integrated analysis of the edaphobiological attributes can be an important tool for soil quality evaluation. We evaluated the soil quality regarding the adopted agriculture practices, using the integrated analysis of physical, chemical and biological attributes. The research was carried out in Ibiúna and Socorro, São Paulo state, Brazil, in organic and conventional production, on small and family farms. Physical, chemical and biochemical parameters were evaluated during 2006 and 2007 in soils under vegetable cultivation, native forest or fallow, taken as reference of natural soil. Agricultural practices data were collected in each production system. The obtained data were submitted to principal component analysis (PCA). There was no pronounced grouping trend according to the production system, organic or conventional, but there was a grouping according to the soil use (forest/fallow land or cultivation). The PCA identified greater degree of similarity between Socorro's cultivation soil and its respective controls compared to Ibiuna's soil, clearly separated according to its use (forest or cropping), indicating better soil management in Socorro. In general, the results allow to conclude that agricultural practices in most of both organic and conventional production systems, caused soil degradation, due to, mainly, intensive soil tillage and no soil mulching, as indicated by the reduction of soil organic matter, microbial biomass, plant emergence and aggregate stability in cultivated areas relative to control ones.

Keywords: physical attributes, chemical attributes, biological attributes, soil, management practices, familiar property.

\section{(Recebido para publicação em 20 de maio de 2010; aceito em 28 de setembro de 2011) (Received on May 20, 2010; accepted on September 28, 2011)}

$\mathrm{O}$ solo é um sistema heterogêneo, descontínuo e estruturado, formado por microhabitats com diferentes características químicas, físicas e biológicas altamente interdependentes, de modo que o ambiente solo pode ser alterado em conseqüência do desmatamento, da remoção de sua camada superficial e intenso revolvimento, com impacto negativo imediato na sua microbiota e em seus processos. Ademais, algumas podem ser medidas, enquanto a mensuração de outras não é possível com o conhecimento atual (Moreira \& Siqueira, 2006).

Considerando-se que o entendimento do conceito de qualidade do solo é tão amplo e compreende o equilíbrio entre os condicionantes geológicos, hidrológicos, químicos, físicos e biológicos (Zilli et al., 2003), Doran \& Parkin (1994) definem a qualidade do solo como "a capacidade do solo funcionar dentro dos limites de um ecossistema, sustentando a produtividade biológica, mantendo a qualidade do meio ambiente e promovendo a saúde dos animais e das plantas".

Indiferente ao sistema de produção utilizado, as tecnologias agrícolas baseadas em monocultivos, a utilização inadequada de agrotóxicos e de fertilizantes químicos e orgânicos, o uso intensivo de máquinas pesadas e a manutenção do solo descoberto, podem afetar, em geral negativamente, a qualidade do solo por mudanças nas propriedades físicas, químicas e biológicas (Valarini et al., 2007). 
Segundo Moreira \& Silva (2006), as variáveis físicas e químicas do solo podem ser utilizadas para mensurar a qualidade do solo. No entanto, a maioria dessas variáveis altera-se mais lentamente, sendo necessário um tempo maior para verificar mudanças significativas em função das práticas agrícolas utilizadas no solo. Por outro lado, segundo Pascual et al. (2000), os indicadores biológicos e bioquímicos do solo foram mais sensíveis às alterações de estresse ou recuperação ecológica, proporcionando informações imediatas e precisas.

A atividade biológica é um importante componente do solo, uma vez que estimula os processos biológicos responsáveis pela formação e manutenção da estrutura física e da fertilidade do solo (Tokeshi et al., 1997). Entre as enzimas do solo de origem microbiana pode-se citar a desidrogenase, que por estar envolvida no processo de transporte de elétrons e acoplada à síntese de ATP, pode ser empregada como medida da atividade microbiana do solo. Esta enzima tem sido utilizada com sucesso como indicador da qualidade do solo (Moreira \& Siqueira, 2006). Trabalhos realizados por Valarini et al. (2002, 2006, 2007) mostraram que os indicadores bioquímicos tais como, a atividade de desidrogenases e o conteúdo de polissacarídeos no solo foram sensíveis para detecção de alterações no manejo agrícola.

Para a avaliação da qualidade de um solo, tem sido postulada a necessidade de identificar parâmetros indicativos e integrados de seu estado de conservação e/ou degradação. Dessa forma, pesquisa realizada sobre análise integrada por Tokeshi et al. (1997); Frighetto \& Valarini (2000); Valarini et al. (2002) recomendam alguns indicadores físicos, químicos e biológicos como, estabilidade de agregados, patógenos de solo, emergência de plantas, atividades de desidrogenases e esterases, biomassa microbiana e polissacarídeos avaliando a estrutura, a fertilidade e as atividades microbiológicas do solo.

A análise conjunta de dados é uma parte essencial em todo experimento. Trabalho relativo ao solo tem apontado carência de conhecimento e subutiliza- ção de métodos multivariados. O modelo estatístico desses métodos considera a correlação entre muitas variáveis analisadas simultaneamente, permitindo a extração de uma quantidade maior de informação (Valarini et al., 2007). A aplicação de métodos quimiométricos (Análise de Componentes Principais ACP e Mapa Auto-Organizável (MAO) na discriminação de práticas de manejo) em análise de solos foi realizada por esses autores no município de Guaíra, SP, utilizando a ACP, cujos resultados permitiram separar parcelas sob manejo convencional do alternativo, principalmente devido ao maior teor de biomassa microbiana e polissacarídeos no solo sob manejo alternativo (Sena et al., 2002).

O objetivo da pesquisa foi avaliar a qualidade do solo em função das práticas agrícolas utilizadas, mediante a análise integrada de atributos precoces e sensíveis, a fim de estabelecer medidas alternativas para um manejo mais adequado do solo.

\section{MATERIAL E MÉTODOS}

A pesquisa foi realizada nas microrregiões de Ibiúna e Socorro, no estado de São Paulo em 2006 e 2007. As propriedades foram selecionadas aos pares, com sistema convencional (SC) e orgânico (SO), em parcerias com a Casa de Agricultura dos municípios, sendo cinco propriedades para cada sistema em cada microrregião. Tanto as localizadas em Socorro como Ibiúna, são pequenas propriedades familiares, com cultivo predominantemente de hortaliças, em áreas variando entre 1-4 ha (Socorro) e 2-10 ha (Ibiúna). As propriedades orgânicas estavam certificadas entre dois a quatro anos (Socorro) e entre três a oito anos (Ibiúna).

O preparo do solo das áreas de produção caracterizou-se basicamente de aração, gradagem e encanteiramento com enxada rotativa, sendo que uma minoria, de 15 a $20 \%$ sob SO, realizou cultivo mínimo e pousio periodicamente. Os tratos fitossanitários no SO se restringiram ao uso do controle biológico e defensivo alternativos. Como adubações dos SO foram utilizados estercos, bokashi, adubos verdes e compostos orgânicos. Já no SC, o solo foi adubado com formulações comerciais de NPK e esterco quando disponível. O controle de pragas e doenças foi baseado no uso de agrotóxicos, no caso do sistema convencional.

Foram feitas duas amostragens anuais de solo em 2006 e em 2007, sendo uma em maio, após estação chuvosa, e a outra em novembro após estação de seca. Em cada propriedade, as análises foram realizadas nos solos sob cultivo de hortaliças de diferentes espécies e em solos sob mata ou pastagem, como áreas de referência, dependendo do local de estudo. As testemunhas foram selecionadas visando representar um estado natural do solo, isto é, sem interferência antrópica do manejo, para melhor expressar os resultados do efeito do manejo sobre as áreas de cultivo.

Os solos das testemunhas (T) e cultivos (C) amostrados nas propriedades para o estudo foram classificados texturalmente nas seguintes classes: franco argiloso arenoso-P1, P2 e P6 de Ibiúna e P6 de Socorro; franco argiloso-P3 e P7 de Ibiúna; argila arenosa-P4, P5, P8, P9 e P10 de Ibiúna e P1, P2, P4, P5, P8, $\mathrm{P} 10$ de Socorro; argila pesada-P3 e P7 de Socorro; argila-P9 de Socorro. Os resultados mostraram ainda que os solos tanto do cultivo como da testemunha para cada propriedade foram classificados na mesma classe textural.

Foram coletadas 10 amostras simples de solo para compor uma amostra composta, na profundidade de $0-20 \mathrm{~cm}$. Para as análises químicas e biológicas, foram coletadas amostras deformadas de solo com trado, enquanto para as análises físicas, foram coletadas amostras indeformadas de solo (anel volumétrico), conforme o atributo a ser determinado. Logo após a coleta, o solo para análise biológica foi acondicionado em caixas de isopor. As amostras foram processadas e encaminhadas aos laboratórios da Embrapa Meio Ambiente (análises físicas e biológicas) e do Instituto Agronômico de Campinas (análises químicas e físicas). Os solos coletados foram submetidos à classificação textural segundo metodologia descrita por Kiehl (1979). Foram estudados os atributos químicos: teor de matéria orgânica (MO), carbono orgânico (C org), $\mathrm{pH}\left(\mathrm{CaCl}_{2}\right), \mathrm{P}, \mathrm{K}$, $\mathrm{Ca}, \mathrm{Mg}, \mathrm{H}+\mathrm{Al}$, soma de bases (SB), 
capacidade de troca catiônica (CTC), saturação por bases (V\%); físicos: condutividade hidráulica saturada (Chs) (Libardi, 2005), capacidade de campo (CC), porosidade de aeração $(\mathrm{Pa})$, compactação (Rs) para resistência do solo (Stolf, 1991) e estabilidade de agregados (Ea) via úmida por meio de DMP. As análises químicas para determinar fertilidade e físicas para estrutura do solo foram baseadas em metodologias descritas em Embrapa (1997) e Raij et al. (2001). As análises biológicas seguiram metodologias descritas em Frighetto \& Valarini (2000), como os atributos carbono da biomassa microbiana $(\mathrm{Bm})$, a atividade de desidrogenases (Desid) e o conteúdo de carbono em polissacarídeos (Poli). Adicionalmente, com parte das amostras indeformadas foram realizados ensaios em casa de vegetação com solos coletados nas propriedades selecionadas nas duas microrregiões visando avaliar o efeito sobre a emergência de feijão e tomate. Paralelamente, as propriedades foram monitoradas quanto às práticas agrícolas e dados climáticos fornecidos pela Casa de Agricultura do respectivo município para subsidiar a interpretação dos resultados.

A análise integrada dos atributos foi realizada por meio da análise de componentes principais (ACP), como forma de selecionar os indicadores de qualidade do solo mais precoces e sensíveis. Para tanto, foi utilizado o valor médio das quatro avaliações de cada atributo do solo realizadas em 2006 e 2007. Esses dados foram analisados no programa MATLABTM, versão 5.2 (The Math Works, Natick, EUA), usando o pacote “PLS Toolbox", versão 2.0 (Eigenvector Technologies, Manson, EUA). Com referência a todas as análises, os dados foram previamente autoescalados (média 0 e variância 1), a fim de que as variáveis contribuíssem igualmente no modelo adotado, independentemente da escala em que foram medidas.

\section{RESULTADOS E DISCUSSÃO}

$\mathrm{Na}$ avaliação da condutividade hidráulica dos solos (Chs), observou-se que os solos apresentaram uma percolação entre 150 e 600 mm/h (Tabela 1), classificada como muito boa segundo

Tabela 1. Indicadores físicos do solo nas áreas testemunhas e de cultivos de propriedades orgânicas e convencionais (soil physical indicators evaluation from control and cultivation areas at organic and conventional production establishments). Ibiúna e Socorro, Embrapa Meio Ambiente, 2006-2007.

\begin{tabular}{|c|c|c|c|c|c|c|}
\hline \multirow{2}{*}{ Região } & \multirow{2}{*}{ Atributos $^{1}$} & \multirow{2}{*}{$\begin{array}{c}\text { Chs } \\
(\mathrm{mm} / \mathrm{h})\end{array}$} & $\mathrm{CC}$ & $\mathbf{P a}$ & \multirow{2}{*}{$\begin{array}{c}\text { Rs } \\
\text { (Kpa) }\end{array}$} & \multirow{2}{*}{$\begin{array}{c}\text { Ea } \\
(\mathrm{mm})\end{array}$} \\
\hline & & & \multicolumn{2}{|c|}{$\mathrm{m}^{3} / \mathrm{m}^{3}$} & & \\
\hline \multirow{4}{*}{ Ibiúna } & $\mathrm{T}^{2} \mathrm{SO}$ & $205,0^{6}$ & 0,4 & 0,2 & 1101,3 & 4,3 \\
\hline & $\mathrm{C}^{3} \mathrm{SO}^{4}$ & 358,4 & 0,3 & 0,2 & 582,7 & 1,9 \\
\hline & $\mathrm{T} \mathrm{SC}^{5}$ & 369,8 & 0,3 & 0,2 & 1192,9 & 3,8 \\
\hline & $\mathrm{C} \mathrm{SC}$ & 324,5 & 0,3 & 0,2 & 504,5 & 1,6 \\
\hline \multirow{4}{*}{ Socorro } & $\mathrm{T} \mathrm{SO}$ & 225,6 & 0,3 & 0,2 & 1417,0 & 3,7 \\
\hline & $\mathrm{C} \mathrm{SO}$ & 380,1 & 0,3 & 0,2 & 691,5 & 3,0 \\
\hline & $\mathrm{T} \mathrm{SC}$ & 276,1 & 0,3 & 0,2 & 1345,9 & 3,5 \\
\hline & $\mathrm{C} \mathrm{SC}$ & 473,9 & 0,3 & 0,2 & 626,4 & 2,6 \\
\hline
\end{tabular}

${ }^{1}$ Atributos avaliados: $\mathrm{Chs}=$ condutividade hidraúlica saturada do solo; $\mathrm{CC}=$ capacidade de campo; $\mathrm{Pa}=$ porosidade de aeração solo; $\mathrm{RS}=$ compactação do solo; Ea= estabilidade de agregados; ${ }^{2} \mathrm{~T}=$ testemunha; ${ }^{3} \mathrm{C}=$ cultivo ${ }^{4} \mathrm{SO}=$ sistema orgânico; ${ }^{5} \mathrm{SC}=$ sistema convencional; ${ }^{6}$ Médias de quatro repetições $\left({ }^{1} \mathrm{Chs}=\right.$ hidraulic conductivity; $\mathrm{CC}=$ water holding capacity; $\mathrm{Pa}=$ soil aeration; $\mathrm{Rs}=$ soil resistance; $\mathrm{Ea}=$ aggregate stability; ${ }^{2} \mathrm{~T}=$ control; ${ }^{3} \mathrm{C}=$ cultivation; ${ }^{4} \mathrm{SO}=$ organic system. ${ }^{5} \mathrm{SC}=$ conventional system. ${ }^{6}$ Means of four replications).

Tabela 2. Indicadores químicos do solo nas áreas testemunhas e de cultivos de propriedades orgânicas e convencionais (soil chemical indicators from control and cultivation areas at organic and conventional production establishments). Ibiúna e Socorro, Embrapa Meio Ambiente, 2006-2007.

\begin{tabular}{|c|c|c|c|c|c|c|c|c|c|c|}
\hline \multirow{2}{*}{ Atributos $^{1}$} & \multirow{2}{*}{$\begin{array}{l}\text { MO }^{1} \\
(\mathrm{~g} / \mathrm{L})\end{array}$} & \multirow{2}{*}{$\begin{array}{c}\mathbf{P H} \\
\mathrm{CaCl}_{2}\end{array}$} & \multirow{2}{*}{$\begin{array}{c}\mathbf{P} \\
(\mathrm{mg} / \mathrm{L})\end{array}$} & $\mathbf{K}$ & $\mathbf{C a}$ & Mg & $\mathbf{H}+\mathbf{A l}$ & SB & CTC & \multirow{2}{*}{$\begin{array}{c}V \\
(\%)\end{array}$} \\
\hline & & & & \multicolumn{6}{|c|}{$\left(\mathrm{mmol}_{\mathrm{c}} \mathrm{dm}^{-3}\right)$} & \\
\hline \multicolumn{11}{|c|}{ Ibiúna } \\
\hline $\mathrm{T}^{2} \mathrm{SO}$ & $46,1^{6}$ & 4,4 & 26,9 & 2,4 & 18,0 & 5,5 & 80,6 & 25,9 & 106,6 & 23,9 \\
\hline $\mathrm{C}^{3} \mathrm{SO}^{4}$ & 33,6 & 5,6 & 174,3 & 4,1 & 59,0 & 12,5 & 30,9 & 75,6 & 106,6 & 67,6 \\
\hline $\mathrm{T} \mathrm{SC}^{5}$ & 44,8 & 4,6 & 54,7 & 2,5 & 22,1 & 6,6 & 77,6 & 31,2 & 108,8 & 30,6 \\
\hline $\mathrm{C} \mathrm{SC}$ & 34,6 & 5,8 & 238,0 & 4,4 & 66,4 & 15,5 & 29,4 & 86,2 & 115,7 & 70,8 \\
\hline \multicolumn{11}{|c|}{ Socorro } \\
\hline T SO & 44,5 & 4,6 & 14,6 & 2,9 & 16,2 & 6,6 & 74,4 & 25,7 & 100,1 & 29,6 \\
\hline C SO & 42,2 & 5,3 & 42,0 & 3,1 & 32,1 & 11,8 & 42,3 & 46,8 & 89,2 & 51,7 \\
\hline $\mathrm{T} \mathrm{SC}$ & 44,1 & 4,8 & 29,0 & 3,3 & 29,1 & 10,4 & 53,1 & 42,8 & 96,1 & 42,5 \\
\hline C SC & 37,0 & 5,0 & 84,5 & 3,7 & 34,8 & 12,5 & 40,3 & 51,0 & 91,4 & 54,3 \\
\hline
\end{tabular}

${ }^{1}$ Matéria orgânica (MO), acidez potencial (H+Al), soma de bases (SB), capacidade de troca catiônica (CTC), índice de saturação por bases (V\%). ${ }^{2}$ Testemunha (T); ${ }^{3}$ Cultivo (C); ${ }^{4}$ Sistema Orgânico (SO); ${ }^{5}$ Sistema Convencional (SC); ${ }^{6}$ Médias de quatro repetições ( $\left({ }^{1}\right.$ soil organic matter, potencial acidity, base content, cationic exchange capacity, percentage of base saturation. ${ }^{2}$ Control; ${ }^{3}$ Cultivation; ${ }^{4}$ Organic system; ${ }^{5}$ Conventional system. ${ }^{6}$ Means of four replications).

relato de Lepsch (1991). Segundo Carvalho et al. (2004), a Chs é uma variável que traduz a facilidade com que a água se movimenta ao longo do perfil de solo. Sua determinação torna-se imprescindível, visto que o movimento da água no solo está diretamente relacionado à retenção e transmissão da água às plantas e, conseqüentemente, pela redução da erosão hídrica (Sing \& Ewing, 2000; Bautista et al., 2004). Já o atributo capacidade de campo (CC), onde as características de retenção de água no solo são expressas por todas as propriedades, os valores se encontraram semelhantes entre 0,3 e $0,4 \mathrm{~m}^{3} / \mathrm{m}$, portanto, não se constituindo neste caso num indicador eficiente. Da mesma forma, a determinação de porosidade de aeração $(\mathrm{Pa})$ apresentou similaridade entre todos os solos avaliados independente do manejo. Segundo Melloni et 
Tabela 3. Indicadores biológicos do solo nas áreas testemunhas e de cultivos de propriedades orgânicas e convencionais (soil biochemical indicators from test control and cultivation areas at organic and conventional production establishments). Ibiúna e Socorro, Embrapa Meio Ambiente, 2006-2007).

\begin{tabular}{|c|c|c|c|c|c|c|}
\hline \multirow{2}{*}{ Região } & \multirow{2}{*}{ Tratamento $^{1}$} & \multirow{2}{*}{$\begin{array}{c}\text { Desid } \\
(\mu \mathrm{L} \text { de } \mathrm{H} / \mathrm{g} \text { solo })\end{array}$} & \multirow{2}{*}{$\begin{array}{c}\text { Polis } \\
\text { (mg/g solo) }\end{array}$} & \multirow{2}{*}{$\begin{array}{c}\mathrm{Bm} \\
\text { (ugC/g solo) }\end{array}$} & EmF & EmT \\
\hline & & & & & \multicolumn{2}{|c|}{$(\%)$} \\
\hline \multirow{4}{*}{ Ibiúna } & $\mathrm{T}^{2} \mathrm{SO}$ & $6,0^{6}$ & 4,1 & 972,0 & 86,6 & 85,3 \\
\hline & $\mathrm{C}^{3} \mathrm{SO}^{4}$ & 6,5 & 2,0 & 718,1 & 74,1 & 70,7 \\
\hline & $\mathrm{T} \mathrm{SC}^{5}$ & 5,6 & 3,9 & 1120,2 & 87,4 & 83,2 \\
\hline & $\mathrm{C} \mathrm{SC}$ & 5,8 & 2,9 & 751,6 & 73,1 & 71,4 \\
\hline \multirow{4}{*}{ Socorro } & T SO & 7,8 & 2,4 & 973,9 & 89,8 & 81,8 \\
\hline & $\mathrm{C} \mathrm{SO}$ & 8,3 & 2,0 & 704,9 & 84,3 & 78,8 \\
\hline & $\mathrm{T} \mathrm{SC}$ & 8,8 & 2,2 & 777,1 & 79,0 & 83,8 \\
\hline & $\mathrm{C} \mathrm{SC}$ & 6,2 & 2,4 & 682,1 & 77,2 & 75,2 \\
\hline
\end{tabular}

${ }^{1}$ Desidrogenase (Desid), conteúdo de polissacarídeo em carbono (Polis), carbono em biomassa microbiana $(\mathrm{Bm})$, emergência em feijão $(\mathrm{EmF})$ e tomate $(\mathrm{EmT}) .{ }^{2}$ Testemunha $(\mathrm{T})$. ${ }^{3}$ Cultivo (C). ${ }^{4}$ Sistema orgânico (SO) e ${ }^{5}$ Sistema convencional (SC). ${ }^{6}$ Médias de quatro avaliações ( ${ }^{1}$ dehydrogenase activity, polysaccharides, carbon microbial biomass, beans and tomato emergence. ${ }^{2}$ Control. ${ }^{3}$ Cultivation. ${ }^{4}$ Organic system. ${ }^{5}$ Conventional system. ${ }^{6}$ Means of four replications).

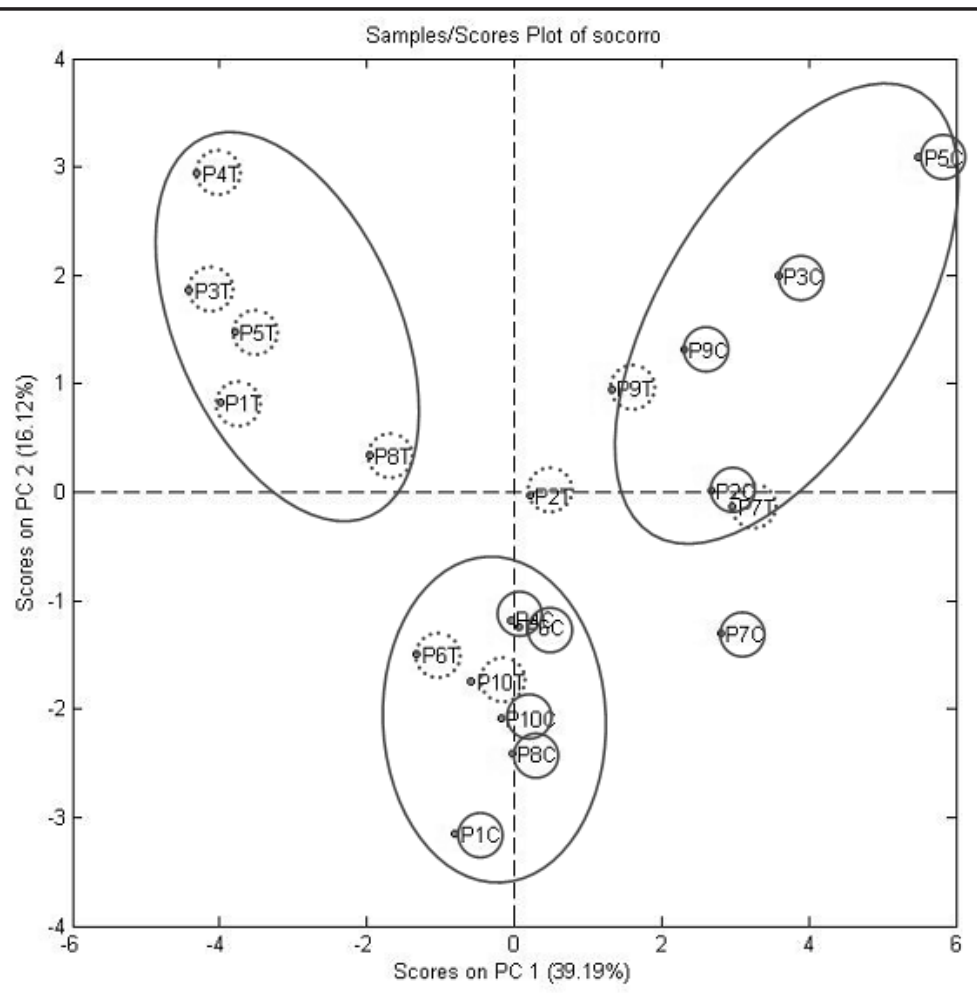

Figura 1. Resultado da Análise de Componentes Principais (ACP) mostrando as duas primeiras componentes para os dados médios de quatro avaliações [results of the Principal Components Analysis (PCA) showing the first two components with four evaluation means]. Socorro, Embrapa Meio Ambiente, 2006-2007.

Propriedade $(\mathrm{P})$, testemunha $(\mathrm{T})$, cultivo $(\mathrm{C})$. Áreas circundadas são locais de cultivo e as tracejadas são testemunhas (production establishments $(\mathrm{P})$ control $(\mathrm{T})$, cultivation $(\mathrm{C})$. Cultivation areas are circled and the dotted areas are control areas).

al. (2008), o conhecimento quantitativo da alteração dos atributos físicos do solo evidenciará seus melhores indicadores de impacto, quando forem comparados diferentes ecossistemas em relação ao ecossistema natural. Na avaliação da compactação do solo (Rs), observou-se que a partir de $10 \mathrm{~cm}$ de profundidade houve aumento gradativo da resistência para todas as amostras coletadas, sem no entanto, ultrapassar $2000 \mathrm{KPa}$, valor limite, que segundo Roselem et al. (1994) e Alvarenga et al. (1996), indica resistência à penetração das raízes das plantas no solo (Tabela 1). Porém, na região de Ibiúna, observou-se que tanto para o SO quanto para o $\mathrm{SC}$, de 0 a 10 $\mathrm{cm}$ de profundidade, há uma resistência do solo menor que $500 \mathrm{KPa}$, indicando intenso revolvimento do solo. Este fato pode ser reforçado pelos baixos valores de estabilidade de agregados (Ea) encontrados nos solos de cultivo nessa região (DMP $=1,9 \mathrm{~mm}$ no $\mathrm{SO}$ contra $1,6 \mathrm{~mm}$ no $\mathrm{SC}$ ), sendo menores que os encontrados em Socorro (3 e 2,6 mm no $\mathrm{SO}$ e SC, respectivamente). Percebe-se ainda maior estabilidade de agregados nos solos sob Sistema Orgânico em relação ao Sistema Convencional e, maior nos solos testemunha que nos de cultivo.

Com relação ao parâmetro $\mathrm{pH}$, o dos solos de cultivo de Ibiúna foi de 5,7 evidenciando baixa acidez enquanto em Socorro foi de 5,1, caracterizando média acidez do solo (Tabela 2). Segundo Bautista et al. (2004) e Melloni et al. (2008), o pH é um importante indicador ligado à acidez do solo e disponibilidade de nutrientes às plantas, pois influencia diretamente na atividade microbiana do solo e no crescimento vegetal.

Uma das variáveis químicas que merece maior destaque, principalmente em Ibiúna, são os altos teores de $\mathrm{P}$ nos solos sob SO (174 mg/L) e SC (238 $\mathrm{mg} / \mathrm{L}$ ) (Tabela 2). Segundo Raij et al. (2001), este parâmetro com teores de até $120 \mathrm{mg} / \mathrm{L}$ são considerados altos e suficientes para obtenção de elevadas produtividades de hortaliças. É conhecido que o excesso de $\mathrm{P}$ no solo pode ocasionar problemas de deficiência de $\mathrm{Zn}$ nas culturas mais sensíveis (Primavesi, 1997). Em solos suscetíveis à erosão pode ocorrer ainda a contaminação dos cursos de água, além de elevar consideravelmente o custo de produção. Em relação a Socorro, os teores de $\mathrm{P}$, tanto no $\mathrm{SO}(42 \mathrm{mg} / \mathrm{L})$ quanto no $\mathrm{SC}(84,5$ $\mathrm{mg} / \mathrm{L}$ ) encontram-se na faixa de média a alta, para hortaliças (Raij et al., 2001), o que também pode ser explicado pela 


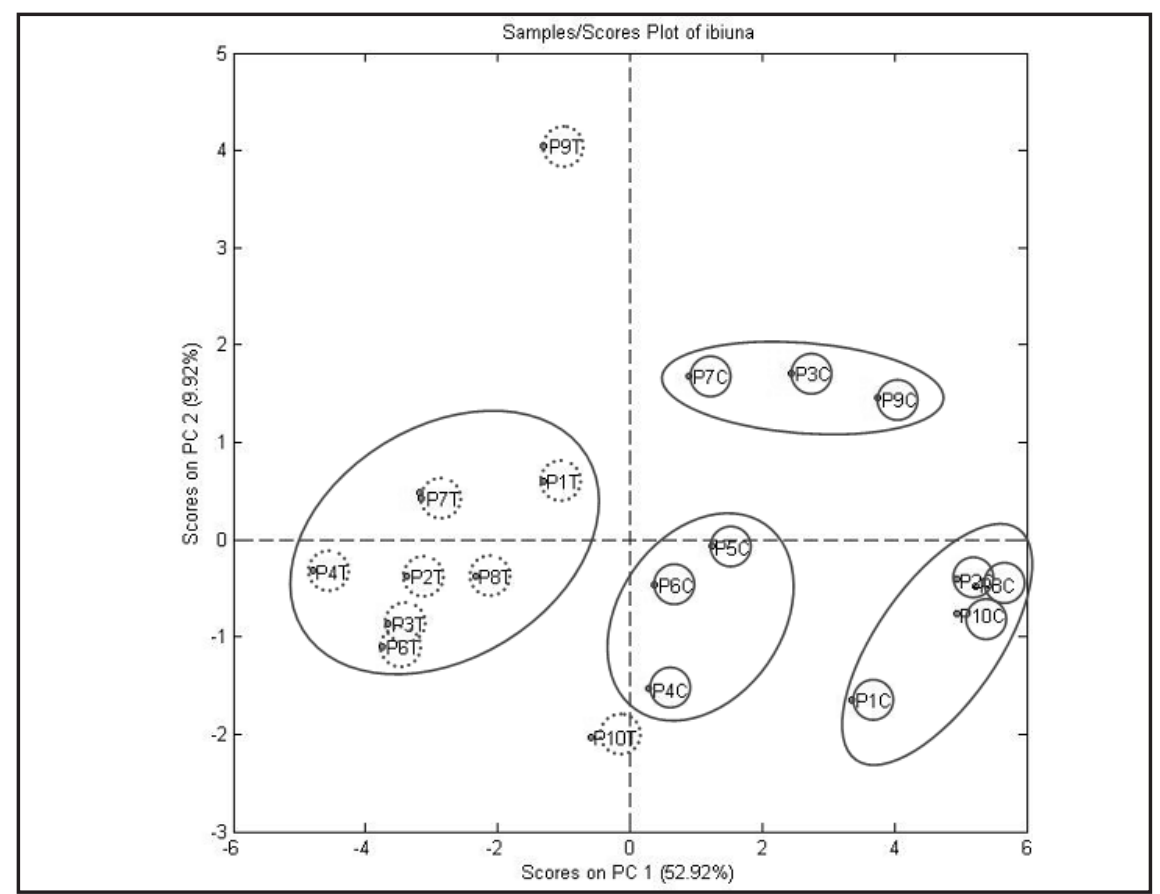

Figura 2. Resultado da Análise de Componentes Principais (ACP) mostrando as duas primeiras componentes para os dados médios de quatro avaliações [results of the Principal Components Analysis (PCA showing the first two components with four evaluation means]. Ibiuna, Embrapa Meio Ambiente, 2006-2007.

Propriedade (P), testemunha (T), cultivo (C). Áreas circundadas são locais de cultivo e as tracejadas são testemunhas (production establishments $(\mathrm{P})$ control $(\mathrm{T})$, cultivation $(\mathrm{C})$. Cultivation areas are circled and the dotted areas are control areas).

menor intensidade de cultivo do solo e quantidade de adubos aplicados.

Os teores de $\mathrm{Ca}, \mathrm{Mg}$ e $\mathrm{K}$ foram considerados elevados em ambas as microrregiões estudadas (Tabela 2). Entretanto, quando se avalia o equilíbrio entre as bases na ocupação da CTC nos solos em Ibiúna, observa-se que tanto no $\mathrm{SO}$ como no $\mathrm{SC}$ a relação $\mathrm{Ca} / \mathrm{Mg}$ foi de 5 , considerada adequada para o cultivo (Primavesi, 2006). Já a relação $\mathrm{Ca} / \mathrm{K}$ calculada foi de 10 e 11 sob SO e SC, respectivamente, valores que segundo a mesma autora, acima do adequado. Para Socorro, o fenômeno se repete para a relação de $\mathrm{Ca} / \mathrm{K}$ em ambos os sistemas, encontrando-se também acima do ideal. Os altos níveis de $\mathrm{Ca}$ no solo podem ocasionar a precipitação do $\mathrm{P}$, através da formação de fosfato tricálcico, altamente insolúvel, além de poder afetar a absorção de outros cátions.

Os efeitos maléficos do desequilíbrio de nutrientes no solo também foram observados nos cultivos do meloeiro por Miranda \& Miranda (1997) em alguns solos de origem calcária no $\mathrm{RN}$ e $\mathrm{CE}$, irrigados com água rica em $\mathrm{Ca}^{2+}$ e com ocorra o desequilíbrio químico do solo com posteriores perdas de produtividade e qualidade (Tabela 2). Além do mais, é reciclador de nutrientes e fontes substitutas de N, $\mathrm{P}$ e Ca.

Em Ibiúna foram encontrados teores médios de $\mathrm{MO}$ nas áreas sob SO $(34 \mathrm{~g} / \mathrm{L})$ e SC (35 g/L), menores que nas áreas testemunhas ( $45 \mathrm{~g} / \mathrm{L})($ Tabela 2$)$. Em Socorro observou-se a mesma tendência de empobrecimento nos solos sob SO (42 $\mathrm{g} / \mathrm{L})$ e SC $(37 \mathrm{~g} / \mathrm{L})$ em relação às áreas testemunhas $(44 \mathrm{~g} / \mathrm{L})$. Observou-se uma redução de $24 \%$ em Ibiúna e de 10\% em Socorro no teor de MO das áreas cultivadas em relação às áreas de mata. A maior redução observada em Ibiúna pode ser atribuída tanto à maior intensidade de uso como à maior fragilidade do solo estimulada pelas suas características intrínsecas. Por outro lado, a perda da matéria orgânica pode estar ligada à própria natureza desta. Para as duas regiões, a redução da $\mathrm{MO}$ nas áreas cultivadas foi menor que a observada por Bayer \& Schneider (1999), os quais constataram redução de até $60 \%$ comparando-se às encontradas nas áreas de mata com as cultivadas convencionalmente. Os valores encontrados, tanto nas áreas de cultivo quanto nas áreas utilizadas como referência, estão abaixo de $50 \mathrm{~g} / \mathrm{L}$, valor médio citado por Raij et al. (2001) para solos cultivados, levando-se em consideração a textura dos solos estudados.

Por exercer efeitos diretos e indiretos sobre as características do solo (físicas, químicas e biológicas) e sobre as plantas, a MO é crucial para a produtividade, especialmente nos trópicos, constituindo-se em alicerce da sustentabilidade agrícola (Moreira \& Siqueira, 2006). A MO desempenha papel fundamental nas funções do solo, mudando constantemente em função do uso desse recurso natural e por isso é considerado um bom indicador de sua qualidade (Doran \& Parkin, 1994). Já a Desid não mostrou diferenciar os solos dos SO e SC em relação aos controles (Tabela 3). Segundo Chu et al. (2007), devido a relação com as atividades energéticas, a Desid possui alta relação com o C da biomassa, respiração basal do solo e $\mathrm{C}$ do solo e, conseqüentemente, com a qualidade e ciclagem da MO, destacando-se como um bom indicador 
da atividade microbiana no solo.

Segundo Melloni et al. (2008), a biomassa microbiana do solo é definida como a parte viva da $\mathrm{MO}$ e, além de ser armazenadora de nutrientes, pode servir como um indicador que mostra sensíveis mudanças no solo, revelando a fragilidade da microbiota à interferências no sistema. Nesse sentido, Mendes et al. (2006) e Arantes \& Melloni (2007), utilizaram uma grande diversidade de indicadores de qualidade para avaliar diferentes ecossistemas, mostrando a extrema sensibilidade microbiana ao manejo do solo, cujo papel esteve sempre associado aos demais atributos físicos, químicos e a própria presença e variação da cobertura vegetal, concordando com Pascual et al. (2000) de que este fator biológico contribuiu favoravelmente à qualidade do solo.

$\mathrm{O}$ empobrecimento do solo em $\mathrm{MO}$ apresentado pela maioria das propriedades de ambas as regiões pode ser explicado pela intensidade de uso do solo, comprometendo os atributos físicos e químicos. Dado o constante uso do solo nas áreas de cultivos e a quase ausente prática de descanso ou de pousio entre uma cultura e outra, observa-se além de uma queda acentuada no teor de $\mathrm{MO}$, menor atividade de biomassa microbiana $(\mathrm{Bm})$. Ainda, com relação aos indicadores biológicos, os resultados avaliados não permitiram verificar diferenças marcantes entre os sistemas de manejo orgânico e convencional em ambas as microrregiões (Tabela 3).

Os resultados das avaliações mostraram que as maiores emergências tanto com feijão (EmF) como com tomate (EmT) foram obtidas em solos das testemunhas, onde ocorreram também maiores valores de Bm, MO e Ea. Porém, em Socorro, em geral, há uma tendência de melhor desempenho da mesma sob solos de $\mathrm{SO}$ em relação aos de $\mathrm{SC}$, o que não parece claro em Ibiúna (Tabela 3).

Com relação às épocas de coleta dos solos, apesar de variações nos dados de temperatura e chuva, não se observou nenhum destaque entre os indicadores de qualidade do solo nas propriedades de SO e SC de ambas as microrregiões avaliadas.

Análise de componentes principais (ACP) - Em Socorro (Figura 1), a componente principal 1 (CP1) separou um grupo formado basicamente por propriedades nas áreas de cultivo $(\mathrm{P} 2 \mathrm{C}$, P3C, P5C, P7T, P9C, P9T), outro com testemunhas (P1T, P3T, P4T, P5T, $\mathrm{P} 8 \mathrm{~T}$ ), e um terceiro grupo com áreas de testemunha e de cultivo. O grupo das testemunhas caracteriza-se por solos com acidez, CTC, teor de matéria orgânica, biomassa microbiana em $\mathrm{C}$, polissacarídeos, compactação do solo e estabilidade de agregados acima da média das áreas estudadas. Já as áreas de cultivo, foram agrupadas por possuírem semelhanças em relação aos teores de $\mathrm{P}$ e K, SB e V\%, além da elevada Chs e $\mathrm{CC}$ dos solos.

Ainda na Figura 1, as propriedades PC6, PC9 e PO10, foram agrupadas com suas respectivas testemunhas, indicando semelhança entre as variáveis analisadas nos solos sob cultivo e sob mata. Tal semelhança pode indicar tanto um bom manejo das áreas de cultivo como, maior tamponamento ou resilência do solo, uma vez que, ainda que utilizados intensivamente, não alteraram substancialmente suas características em relação ao solo de referência (mata).

Em Ibiúna (Figura 2), utilizando duas componentes principais foi possível explicar $63 \%$ da variância total. Neste caso, a separação entre as áreas de cultivo e testemunha foi ainda mais evidente, agrupando valores de escores negativos no PC1 para todas as áreas testemunhas e positivos para áreas de cultivo. A componente principal 1 (PC1) separa o grupo formado por P1C, P2C, $\mathrm{P} 8 \mathrm{C}$ e $\mathrm{P} 10 \mathrm{C}$ dos demais com valores de escores em PC1 bastante positivos. O PC2 separa o grupo formado por (P3C, P7C e P9C) das demais áreas de cultivo, com valores de escores em PC2 positivos. As variáveis responsáveis pela separação entre as testemunhas e cultivos são: Ea, EmT, EmF, Poli, Rs, $\mathrm{Bm}, \mathrm{MO}, \mathrm{CC}$ e H+Al. Observou-se ainda a separação das áreas de cultivo em três grupos, P4C, P5C e P6C; P1C, P2C, P8C e P10C e; P3C, P7C e P9C com CTC; V\%, SB, P, K, Mg e Desid, As, como as variáveis responsáveis, respectivamente.

Observou-se que tanto o agrupamento (Socorro) como a separação (Ibiúna) das áreas de cultivo foram explicados principalmente pelas variáveis químicas (V\%, SB, P e K). Sendo solos tropicais, em geral, caracterizados por serem bastante rasos, com $\mathrm{pH}$ ácido e extremamente pobre, favorecem o desbarrancamento e a erosão, eventos bastante comuns na mata atlântica onde se encontram essas microregiões. Spera (1995) verificou que os solos de mata apresentaram maiores porcentagens de argila dispersa em água, macro e microporosidade, maiores teores de $\mathrm{MO}$ e maior diâmetro médio de agregados na camada superficial, sendo também, mais profundos que os solos de cultivo, com maior capacidade de armazenamento de água no perfil.

Entre as técnicas de análise de agrupamento, freqüentemente utilizada para o melhor aproveitamento dos dados, mantendo a maioria ou todas as informações originais, está a análise de componentes principais. Nesse sentido, já existem vários exemplos de aplicação dessa metodologia em trabalhos para separação das áreas degradadas ou em processos de degradação do ponto de vista microbiológico ou bioquímico, químico e físico, espacialmente daquelas não degradadas (Melloni et al., 2008). Valarini et al. (2007) relataram em trabalho realizado com solos de propriedades orgânicas (SO) e convencionais (SC), que a análise de componentes principais permitiu concluir que há maior grau de similaridade entre os solos sob SO e aqueles das bases referenciais (matas), com respeito aos indicadores químicos ( $\mathrm{V} \%, \mathrm{pH}$, teores de $\mathrm{Mg}, \mathrm{Ca}$ e $\mathrm{Mn}$ ) e biológicos (polissacarídeos, desidrogenase e FDA - Hidrólise de Acetato de Fluoresceína). Também, constataram que esses indicadores biológicos e os químicos (carbono orgânico e nitrogênio total) estão positivamente relacionados com o $\mathrm{SO}$, a mata nativa e a pastagem (controles). Em contrapartida, a saturação por bases (V\%), pH, teores de $\mathrm{Mn}, \mathrm{Mg}$ e $\mathrm{Ca}$, bem como a razão de dispersão estão inversamente relacionadas ao SO. Esses autores confirmam a relevância da análise integrada de indicadores edafobiológicos ligados ao manejo do solo, constituindo-se em importante ferramenta para avaliar níveis de sustentabilidade do agroecossistema e detectar pontos críticos para a devida 
correção de manejo.

No presente estudo (Figuras 1 e 2), apesar de identificado melhor desempenho dos indicadores de qualidade do solo Rs, Ea, P e Desid no $\mathrm{SO}$ e do indicador conteúdo em Poli no SC, a análise de componentes principais (ACP) não identificou uma tendência nítida de agrupamento de acordo com o sistema de produção (SC ou SO). No entanto, de uma forma geral, observa-se agrupamento em função do uso do solo, ou seja, mata/pousio ou cultivo. A ACP identificou ainda maior grau de similaridade entre os solos sob cultivo em Socorro e suas respectivas testemunhas que os solos de Ibiúna, nitidamente separados em função do seu uso (mata ou cultivo) e indicando melhor manejo e conservação dos mesmos em Socorro.

Os resultados indicaram ainda, que tanto para o SC quanto para o $\mathrm{SO}$, as práticas agrícolas utilizadas na maioria das propriedades, tais como a preparação mecanizada e intensiva e a falta de cobertura do solo, a ausência de rotação de culturas com diferentes sistemas radiculares, e o baixo uso de adubação verde proporcionaram degradação do solo. Essa constatação é devida à redução nos teores de $\mathrm{MO}, \mathrm{C}$ em biomassa microbiana, emergência de plântulas e estabilidade de agregados nas áreas sob cultivo em relação aos controles, necessitando adequar medidas de manejo do solo direcionadas para a sustentabilidade dos agroecossistemas em ambas as microrregiões.

\section{AGRADECIMENTOS}

À FAPESP pelo financiamento parcial da pesquisa.

\section{REFERÊNCIAS}

ALVARENGA RC; COSTA LM; MOURA FILHO W; REGAZZI AJ. 1996. Crescimento de raízes de leguminosas em camadas de solo compactadas artificialmente. Revista Brasileira de Ciência do Solo 20: 319-326.

ARANTES D; MELLONI R. 2007. Efeito da declividade em atributos bioquímicos de solos da Reserva Biológica da Serra dos Toledos, Itajubá-MG. In: JORNADA DE INICIAÇÃO CIENTÍFICA, 14. Resumos... Itajubá: INIFEI. (CD-ROM)
BAUTISTA CA; ETCHEVERS B; CASTILLO RF; GUTIÉRREZ C. 2004. La calidad del suelo y sus indicadores. Ecosistemas 13: 96-105. Disponível em http://www.aeet.org/ ecosistemas/042/revision2.htm. Acessado em 10 de novembro de 2007.

BAYER C; SCHNEIDER NG. 1999. Plantio direto e o aumento no conteúdo de matéria orgânica do solo em pequenas propriedades rurais no município de Teutônia. Ciência Rural 29: $165-166$.

CARVALHO R; GOEDERT WJ; ARMANDO MS. 2004. Atributos físicos de qualidade de um solo sistema agroflorestal. Pesquisa Agropecuária Brasileira 39: 1153-1155.

CHU H; LIN X; FUJII T; MORIMOTO S; YAGI K; HU J; ZHANG J. 2007. Soil microbial biomass, dehydrogenase activity, bacterial community structure in response to longterm fertilizer management. Soil Biol. Biochem 39: 2971-2976.

DORAN JW; PARKIN TB. 1994. Defining and assessing soil quality. In: DORAN JW; COEMAN DC; BEZDICEK DF; STEWART BA. Defining soil quality for sustainable environment. Madison: Soil Science Society of America. p. 3-21. (SSSA Special Publication, v.35).

EMBRAPA. Serviço Nacional de Levantamento e Conservação de Solos. 1997. Manual de métodos de análise do solo. $2^{\mathrm{a}}$ ed. Rio de Janeiro: EMBRAPA-CNPS. 212 p. (EmbrapaCNPS. Documentos, 1).

FRIGUETTO RTS; VALARINI PJ. 2000. Indicadores biológicos e bioquímicos da qualidade do solo: manual técnico. Jaguariúna: Embrapa Meio Ambiente. 198p. (Embrapa Meio Ambiente. Documentos, 21).

KIEHL EJ. 1979. Manual de edafologia - Relação solo planta. São Paulo: Ed. Agronômica Ceres. 262p.

LEPSCH IF (Coord) 1991. Manual para levantamento utilitário do meio físico e classificação de terras no sistema de capacidade de uso. $4^{\mathrm{a}}$ aproximação, $2^{\mathrm{a}}$ impressão rev. Campinas: Sociedade Brasileira de Ciência do Solo, 1991. 175 p.

LIBARDI PL. 2005. Dinâmica da água no solo. São Paulo, Ed. EDUSP, 335p.

MALAVOLTA E; VITTI GC; OLIVEIRA SA. 1997. Avaliação do estado nutricional das plantas: princípios e aplicações. 2.ed. Piracicaba: POTAFOS. 319 p.

MELLONI R; MELLONI EGP; ALVARENGA MIN. 2008. Indicadores da qualidade do solo. Informe Agropecuário 29: 17-29.

MENDES FG; MELLONI EGP; MELLONI R. 2006. Aplicação de atributos físicos do solo no estudo da qualidade de áreas impactadas, em Itajubá-MG. Cerne 12: 211-220.

MIRANDA JCC; MIRANDA LN. 1997. Tecnologias: Micorriza aumenta a produtividade das plantas e a eficiência dos insumos. Planaltina: EMBRAPA-CPAC. Disponível em http://www.cpac.embrapa.br/ tecnologias/micorriza.html. Acessado em 16 de novembro de 2008.

MOREIRA FMS; SIQUEIRA JO. 2006.
Microbiologia e bioquímica do solo. 2.ed. atual. ampl. Lavras: UFLA. 625p.

PASCUAL JA; GARCIA C; HERNANDEZ T; MORENO JL; ROS M. 2000. Soil microbial activity as a biomarker of degradation and remediation processes. Soil Biology \& Biochemistry 32: 1877-1883.

PRIMAVESI A. 1997. Agroecologia: ecosfera, tecnosfera e agricultura. São Paulo: Nobel. 199p.

PRIMAVESI A. 2006. Cartilha do solo. São Paulo: Fundação Mokiti Okada. 118p.

RAIJ B; ANDRADE JC; QUAGGIO JAA. 2001. Análise química para avaliação da fertilidade de solos tropicais. Campinas: Instituto Agronômico. 285p.

ROSOLEM CA; ALMEIDA ACS; SACRAMENTO LVS. 1994. Sistema radicular e nutrição da soja em função da compactação do solo. Bragantia 53: 259-266.

SENA MM; FRIGHETTO RTS; VALARINI PJ; TOKESHI H; POPPI RJ 2002. Discrimination of management effects on soil parameters by using principal component analysis: a multivariate analysis case study. Soil \& Tillage Research, 67: 171-181.

SINGER MJ; EWING S. 2000. Soil quality. In: SUMMER ME. (ed). Handbook of soil science. Boca Raton: CRC Press. p. 271-298.

SPERA ST. 1995. Inter-relações entre propriedades físico-hídricos do solo e a ocorrência de vegetação de mata e campo adjacentes no Alto do Rio Grande (MG). Lavras: UFV. 78p (Tese mestrado).

STOLF, R. 1991. Teoria e teste experimental de fórmulas de transformação dos dados de penetrômetro de impacto em resistência do solo. Revista Brasileira de Ciência do Solo 15: 229-235.

TOKESHI H; ALVES MC; SANCHES AB; HARADA DY. 1997. Control of Sclerotinia sclerotiorum with effective microorganisms. Summa Phytopathology 23: 146-154.

VALARINI PJ; DIAZ ALVAREZ MC; GASCÓ JM; GUERRERO F; TOKESHI H. 2002. Integrated evaluation of soil quality after the incorporation of organic matter and microorganisms. Brazilian Journal of Microbiology 33: 53-58.

VALARINI PJ; FRIGHETTO RTS; SCHIAVINATO RJ; CAMPANHOLA C; SENA MM; BALBINO TL; POPPI RJ. 2007. Análise integrada de sistemas de produção de tomateiro com base em indicadores edafobiológicos. Horticultura Brasileira 25: 60-67.

VALARINI PJ; FRIGHETTO RTS; TOKESHI H. 2006. Impacto ambiental do manejo agrícola sobre o meio biótico do solo em áreas irrigadas. In: VALARINI PJ; LUIZ AJB (ed). Impacto ambiental da agricultura irrigada em Guaíra-SP. Jaguariúna: Embrapa Meio Ambiente. p.155-173.

ZILLI EJ; RUMJANEK NG; XAVIER GR; COUTINHO HLC; NEVES MCP. 2003. Diversidade microbiana como indicador de qualidade do solo. Cadernos de Ciência \& Tecnologia 20: 391-411. 